\title{
Glutamatergic Signaling by Mesolimbic Dopamine Neurons in the Nucleus Accumbens
}

\author{
Fatuel Tecuapetla, ${ }^{1}$ Jyoti C. Patel, ${ }^{2}$ Harry Xenias, ${ }^{1}$ Daniel English, ${ }^{1}$ Ibrahim Tadros, ${ }^{1}$ Fulva Shah, ${ }^{1}$ Joshua Berlin, ${ }^{3}$ \\ Karl Deisseroth, ${ }^{4}$ Margaret E. Rice, ${ }^{2}$ James M. Tepper, ${ }^{1}$ and Tibor Koos $^{1}$ \\ ${ }^{1}$ Center for Molecular and Behavioral Neuroscience, Rutgers University, Newark, New Jersey 07102, ${ }^{2}$ Departments of Neurosurgery and Physiology and \\ Neuroscience, New York University School of Medicine, New York, New York 10016, ${ }^{3}$ Department of Pharmacology and Physiology, New Jersey Medical \\ School, Newark, New Jersey 07103, and ${ }^{4}$ Department of Bioengineering, Stanford University, Stanford, California 94305
}

Recent evidence suggests the intriguing possibility that midbrain dopaminergic (DAergic) neurons may use fast glutamatergic transmission to communicate with their postsynaptic targets. Because of technical limitations, direct demonstration of the existence of this signaling mechanism has been limited to experiments using cell culture preparations that often alter neuronal function including neurotransmitter phenotype. Consequently, it remains uncertain whether glutamatergic signaling between DAergic neurons and their postsynaptic targets exists under physiological conditions. Here, using an optogenetic approach, we provide the first conclusive demonstration that mesolimbic DAergic neurons in mice release glutamate and elicit excitatory postsynaptic responses in projection neurons of the nucleus accumbens. In addition, we describe the properties of the postsynaptic glutamatergic responses of these neurons during experimentally evoked burst firing of DAergic axons that reproduce the reward-related phasic population activity of the mesolimbic projection. These observations indicate that, in addition to DAergic mechanisms, mesolimbic reward signaling may involve glutamatergic transmission.

\section{Introduction}

Indirect evidence suggests that mesolimbic dopaminergic (DAergic) neurons may release glutamate as a cotransmitter to communicate with target neurons in the forebrain. This possibility was first suggested on the basis of colocalization of tyrosine hydroxylase (TH) and glutamate in midbrain DAergic neurons as well as autaptic glutamatergic EPSCs in DAergic neurons in midbrain cell culture (Sulzer et al., 1998; Bourque and Trudeau, 2000). EPSPs and EPSCs were also seen in accumbens spiny neurons in ventral tegmental area (VTA)-accumbens cocultures (Sulzer et al., 1998; Bourque and Trudeau, 2000; Joyce and Rayport, 2000; Sulzer and Rayport, 2000) and in nucleus accumbens spiny neurons in response to chemical or electrical stimulation of the VTA in acute brain slices. These responses were suggested to originate from activation of the VTA DAergic projection neurons (Chuhma et al., 2004, 2009).

However, the existence of glutamatergic signaling by DAergic neurons in vivo remains extremely controversial for several reasons. DAergic neurons in vivo lack local axon collaterals (Juraska et al., 1977; Tepper et al., 1987). Furthermore, the nonspecific nature of electrical or chemical stimulation precludes the selective activation of DAergic efferents. Non-

Received Jan. 16, 2010; revised Feb. 27, 2010; accepted March 31, 2010.

This research was supported by Busch Biomedical Research Grants of Rutgers University NS052370 to T.K., NSO34865 to J.M.T., CONACyT to F.T., and NS036362 to M.E.R. We thank D. Pare for discussions, and D. Pare and M. Way for reading of the manuscript.

Correspondence should be addressed to Dr. Tibor Koos, Center for Molecular and Behavioral Neuroscience, Rutgers University, 197 University Avenue, Newark, NJ 07102. E-mail: tibkoos@yahoo.com.

DOI:10.1523/JNEUROSCI.0265-10.2010

Copyright $\odot 2010$ the authors $\quad 0270-6474 / 10 / 307105-06 \$ 15.00 / 0$
DAergic neurons in the VTA that express the vesicular glutamate transporter 2 (VGluT2), a marker for glutamatergic neurons, have been conclusively demonstrated (Yamaguchi et al., 2007), and both ascending and descending fibers of passage, including glutamatergic projections, pass around and through the VTA. Thus, glutamatergic responses in the nucleus accumbens following midbrain stimulation (Chuhma et al., 2004, 2009) may have originated, at least in part, from stimulation of glutamatergic projections originating from sources other than DAergic neurons.

Most importantly, several in situ hybridization studies failed to detect VGluT isoforms in more than a negligible fraction of midbrain $\mathrm{TH}^{+}$neurons in adult animals (Gras et al., 2002; Yamaguchi et al., 2007; Mendez et al., 2008; Bérubé-Carrière et al., 2009). Moreover, a recent immunocytochemical and in situ hybridization study has shown that the expression of VGluT2, the only VGluT isoform associated with DAergic neurons, is developmentally regulated and cannot be detected after postnatal day (PD) 90 (Bérubé-Carrière et al., 2009). Thus, the preponderance of molecular evidence indicates that adult DAergic neurons do not express VGLuT isoforms in vivo (for contrary evidence, see Chuhma et al., 2009). Since VGluT transporters are the only molecules currently known to sequester glutamate in synaptic vesicles, their absence suggests that the cell culture observations may not be representative of the normal functioning of DAergic neurons in vivo. Although the molecular evidence seriously challenges the notion that DA neurons use glutamate as a cotransmitter in vivo, this possibility cannot be excluded on this basis alone because glutamate release might be supported by low levels of VGLuT expression or by some other unidentified mechanism. 
Because of these considerations, unambiguous demonstration of glutamate release by DAergic neurons requires an experimental approach that allows specific and selective activation of DAergic pathways in a preparation that preserves the physiological state of the DAergic axons and their postsynaptic targets.

\section{Materials and Methods}

Viral-mediated gene transfer. Channelrhodop$\sin 2$ (ChR2)-yellow fluorescent protein (YFP) was expressed from a previously described Crelox-controlled transgene (Tsai et al., 2009) delivered with a serotype- 2 adenoassociated virus $(A A V-2)$ viral vector introduced into BAC transgenic TH-Cre [Tg(Th-cre)12Gsat; Gene Expression Nervous System Atlas] or heterozygotic DA transporter (DAT)-internal ribosome entry site (IRES)-Cre mice (B6.SJL-Slc6a $3^{\text {tm1.1( (cre) Bkmn }}$, J, Jackson Laboratories). A $1 \mu$ l, high-titer (1.4* $10^{13}$ viral genome $/ \mathrm{ml}$ ) AAV-2 viral stock solution (Vector Biolabs) was pressure injected into the VTA at the following coordinates: anteroposterior, midway between $-3.5 \mathrm{~mm}$ from bregma and $0.4 \mathrm{~mm}$ from lambda; length, 1.65 $\mathrm{mm}$; and depth, $4.45 \mathrm{~mm}$ below the surface of the brain inclined by $17^{\circ}$ toward the midline. Slices were prepared 9-110 d postinjection.

Slice preparation, and whole-cell and cellattached patch recording. Surgical procedures were performed in accordance with the $\mathrm{Na}$ tional Institutes of Health Guide to the Care and Use of Laboratory Animals and with the approval of the Rutgers University Institutional Animal Care and Use Committee. Mice of both sexes were used and were 90-306 d old when killed. Horizontal slices were prepared at the level of the anterior part of the anterior commisure. Details of slice preparation, and recording methods are described by Tecuapetla et al. (2009). The NMDA receptor component of the EPSCs was isolated by recording the average postsynaptic response at $+50 \mathrm{mV}$ holding potential and subtracting from this the average response recorded after blocking NMDA receptors with APV $(50 \mu \mathrm{M})$.

Optical stimulation. Optical stimuli were delivered from 100 or $200 \mu \mathrm{m}$ multimode optical fibers (THORLABS) coupled to a $150 \mathrm{~mW}, 453$ $\mathrm{nm}$, diode-pumped, solid-state laser (OEM Laser Systems). The laser beam was in most cases aimed at least a distance of $150 \mu \mathrm{m}$ posterior and lateral from the recording site. Maximal intensity light pulses failed to produce any detectable postsynaptic responses in spiny projection neurons (SPNs) in control animals $(n=3$, data not shown).

Voltammetry. Fast-scan cyclic voltammetry was performed as described in detail in Chen and Rice (2001) and Patel et al. (2009). Briefly, recordings were made with a Millar voltammeter using $7-\mu \mathrm{m}$-diameter, $30-70-\mu \mathrm{m}$-long carbon fiber microelectrodes scanning from $-0.7 \mathrm{~V}$ to $+1.3 \mathrm{~V}$ (vs $\mathrm{Ag} / \mathrm{AgCl}$ ) at a rate of $800 \mathrm{~V} / \mathrm{s}$, and sampling at $100 \mathrm{~ms}$ intervals. Identification of released DA was based on voltammograms displaying the single oxidation and reduction peak potentials that define the voltammetric signature of DA (see Fig. $2 D$ ).

The combined electrical and optical stimulation experiments were designed to determine whether there was a difference between the release probability and/or short-term plasticity of transmission from

B
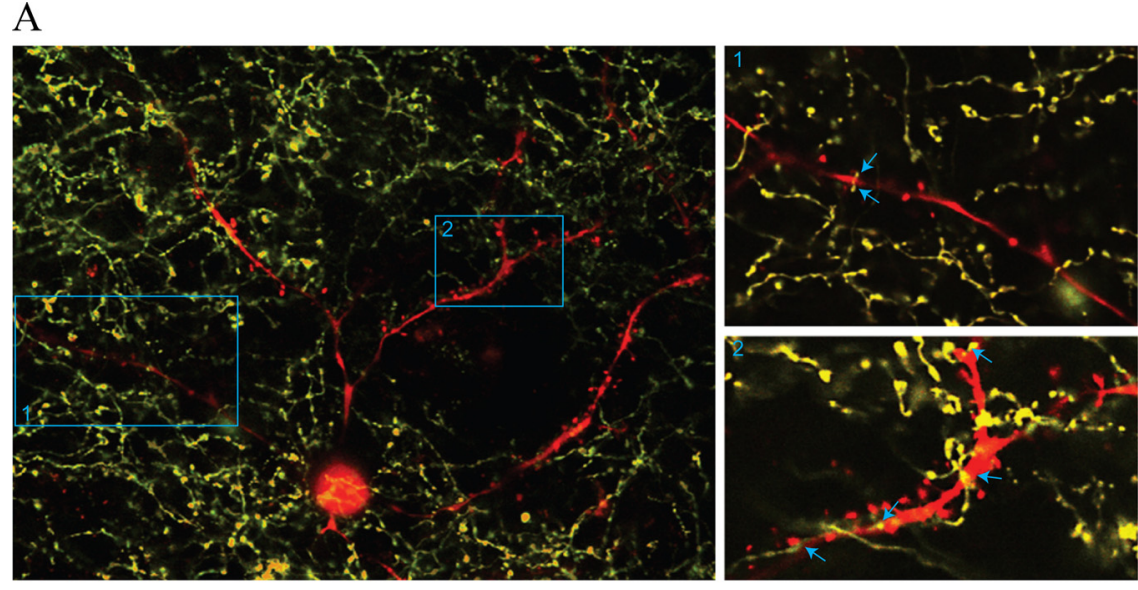

$\mathrm{D}$
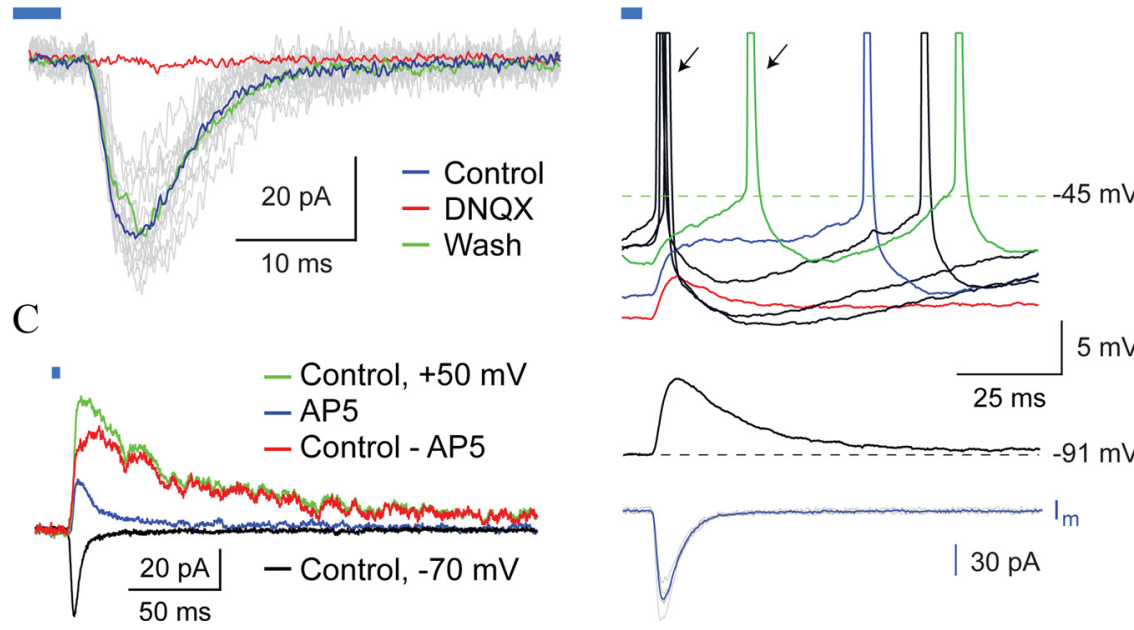
$5 \mathrm{mV}$
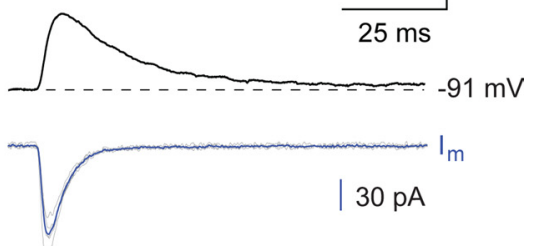

igure 1. Functional glutamatergic transmission by DAergic axons in the nucleus accumbens. $\boldsymbol{A}$, Left, composite confoca . The limited thickness of the displayed optical section ( $\sim 4 \mu \mathrm{m}$ in depth) is to (he extremely dense DAergic axon arborization. Right, Confocal microscopic images (single optical putative close oppositions between the SPN and DAergic axons. $\boldsymbol{B}$, AMPA receptor-mediated EPSCs in a nucleus

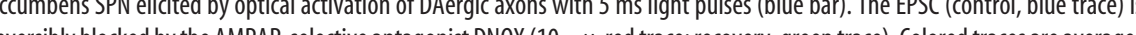
of 10 EPSCs, individual predrug control responses are shown in gray. C, NMDAR-mediated EPSC elicited with the same stimuli as in $B$ in an SPN in the accumbens $\left(V_{h}=+50 \mathrm{mV}\right)$. The average EPSC recorded in the control condition (green), in the presence of the selective NMDAR antagonist APV (blue), and the difference in the two traces corresponding to the NMDAR EPSC (red) are shown. depolarized above $-52 \mathrm{mV}$ in current clamp mode. Traces represent successively larger current injections. Bottom, Corresponding EPSCs in voltage clamp (blue, average; gray, individual EPSCs; $V_{m}=-70 \mathrm{mV}$ ).

DA terminals when stimulated with conventional electrical stimulation or by activating ChR2 currents in axons with optical stimulation. Under the conditions used here $\left(2 \mathrm{~mm}\left[\mathrm{Ca}^{2+}\right]_{\mathrm{e}}\right)$ extracellular DA accumulation exhibits significant short-term plasticity, the magnitude and direction of which reflect in part the release probability and are not solely determined by the activity and saturation of DA reuptake. Because of these characteristics, the rate of extracellular DA accumulation during a stimulation train will reflect the evolution of the release probability over consecutive stimuli, and, therefore, this measure can be used for the comparison of release probabilities under different stimulation conditions. To exclude the possibility that the observed difference in accumulation rates is simply a reflection of different absolute concentrations of DA in the extracellular space leading to different levels of saturation of the reuptake carrier the intensity of electrical stimuli $(0.3-0.5 \mathrm{~mA})$ was adjusted to approxi- 
A
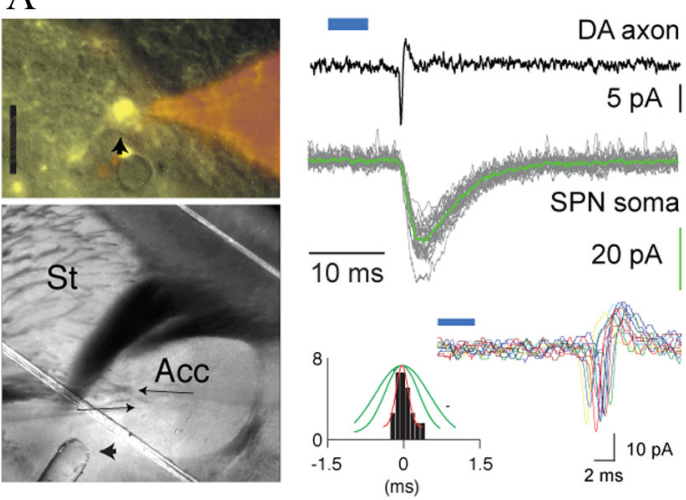

$\mathrm{C}$
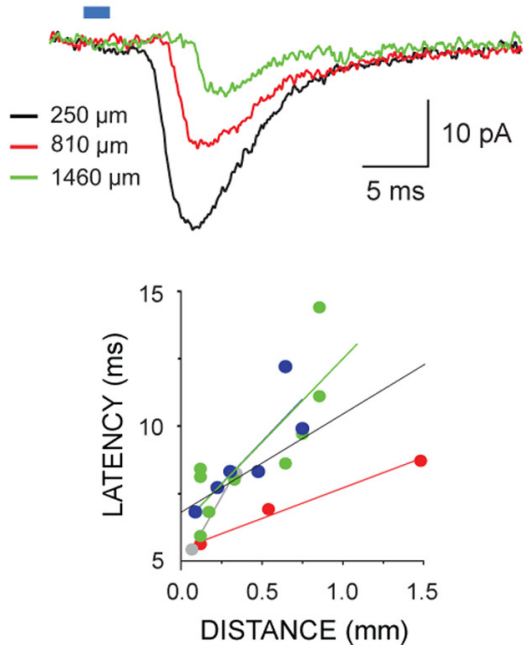

B
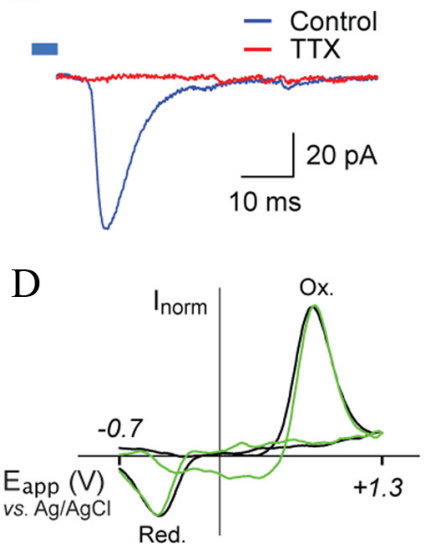

$\mathrm{E}$

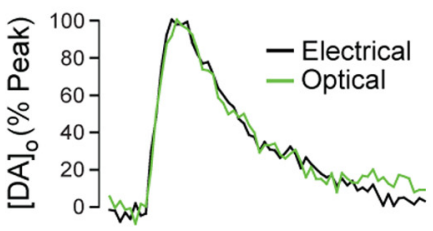

IIIII

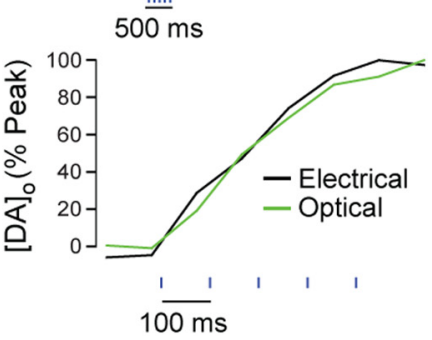

Figure 2. Optically induced synaptic release is physiological. $\boldsymbol{A}$, Reliable and temporally precise control of axonal firing with optical stimulation. Top left, Epifluorescence image of a DAergic, YFP ${ }^{+}$axon bleb (yellow structure, arrowhead) patched with a pipette filled with Alexa Fluor 594 (red). Bottom left, differential interference contrast image of the recording area illustrates pipette positions (arrows) for recordings shown on the right. Optical fiber is seen at arrowhead. Top right, Averaged spikes in a DAergic axon evoked by a 5 ms light pulse (blue bar) recorded in cell-attached patch mode (black trace). Averaged whole-cell EPSCs (green traces) recorded in a nearby SPN show that the onset of the EPSC is coincident with the axonal response. Gray traces are individual EPSCs. Bottom right, Individual action potentials (colored traces, $n=12$ ) recorded in one axon illustrate the reliability of the response and the limited variability of the latency to spike. Bottom middle, Histogram of the distribution of spike delays normalized to the mean and fitted with a Gaussian function are shown for this example (bottom histogram and curve in red). Green curves are similar Gaussian fits to spike delay distributions measured in two other axons. $\boldsymbol{B}$, The optically evoked EPSC (average, blue trace) is blocked by TTX ( $1 \mu \mathrm{m}$, red trace). $\boldsymbol{C}$, The EPSC is elicited by propagating action potentials. Top traces show EPSCs in one SPN in response to optical stimuli delivered at increasing distances from the neuron as indicated on the left. Bottom graph shows latency-distance relationships for four neurons. Lines are linear fits; the fit to the combined data are shown in black. Note the consistent effect of distance on latency. D. Cyclic voltammograms obtained with FSCV in a DA standard ( $1 \mu \mathrm{M}$, black trace) and at the peak of the FSCV current response to optical stimulation (St) in the core of the accumbens (Acc) (green trace). Note the identical positions of the oxidation (0x.) and reduction (Red.) peaks, and the similarity of the waveforms indicating that the detected substance is DA. $\boldsymbol{E}$, Time course of the extracellular DA concentration in the accumbens shell (normalized to peak) during electrical (black trace) and optical (green trace) train stimulation (five stimuli, $10 \mathrm{~Hz}$, blue ticks). The raising phase of the response (top graph) during the first $700 \mathrm{~ms}$ is shown at higher resolution on the bottom. Note the nearly identical rates of extracellular DA accumulation in response to electrical and optical stimulation.

mate the same peak $[\mathrm{DA}]_{e}$ as achieved with optical stimulation. Dopamine concentration ratios were calculated from measurements taken at 100 and $300 \mathrm{~ms}$ after stimulus onset. These time points were chosen to allow comparison of the early and the late parts of the DA concentration profile during train stimulation.

Dopamine depletion. Mice were injected intraperitoneally with 5 $\mathrm{mg} / \mathrm{kg}$ the vesicular monoamine transporter inhibitor reserpine $24 \mathrm{~h}$ before dissection, and with 400 and $200 \mathrm{mg} / \mathrm{kg}$ the tyrosine hydroxylase inhibitor $\alpha$-methyl-para-tyrosine (AMPT) 4 and $2 \mathrm{~h}$ before dissection, respectively. Slices were preincubated for $>1 \mathrm{~h}$ in reserpine $(1 \mu \mathrm{M})$ and AMPT $(30 \mu \mathrm{M})$, and recordings were obtained in the presence of these drugs. This procedure has been demonstrated to result in complete de- pletion of DA (Sullivan et al., 2008), and its efficacy was verified using fast-scan cyclic voltammetry (FSCV) (see Fig. 4B).

\section{Results}

Here, we used a ChR2-based optogenetic method to achieve selective activation of DAergic axons to test the hypothesis that DAergic neurons communicate with postsynaptic targets using glutamatergic transmission. ChR2-YFP fusion protein was specifically targeted to DAergic neurons using viral-mediated transfer of a previously described transgene in which the inverted orientation of a doubly floxed ChR2-YFP coding sequence relative to an Ef- $1_{\text {a }}$ promoter renders ChR2-YFP expression absolutely dependent on Cremediated inversional recombination and consequently on the presence of Cre (Tsai et al., 2009). By introducing this construct in the ventral tegmental area of transgenic mice engineered to express Cre in $\mathrm{TH}$ or DA transporter (DAT)-containing neurons (TH-Cre and DAT-Cre mice, respectively), ChR2-YFP expression could be restricted to $\mathrm{TH}^{+}$or $\mathrm{DAT}^{+}$midbrain afferents to the nucleus accumbens that are conclusively DAergic.

The existence of glutamatergic postsynaptic responses to synchronous focal activation of DAergic fibers with laser light pulses of 2-5 ms duration were investigated using in vitro whole-cell recording from SPNs in horizontal brain slices of the nucleus accumbens obtained from adult (PD 90-306) mice. SPNs were identified using morphological and electrophysiological criteria (Tecuapetla et al., 2009). Single-pulse activation of DAergic axons near the recorded neurons elicited fast inward currents in all tested cells $(n=$ 65; 14 in TH-Cre and 51 in DAT-IRESCre mice) exhibiting an average peak amplitude of $24 \pm 3 \mathrm{pA}$ (range, 6-87 pA; $n=26$ ), a rise time of $2.2 \pm 0.1 \mathrm{~ms}$, and a decay time constant of $5.98 \pm 0.3 \mathrm{~ms}$ $(n=26)$ (Fig. $1 A, B)$. The response was reversibly blocked by the selective AMPA receptor (AMPAR) antagonist DNQX (10 $\mu \mathrm{M}, n=6$ ) and had a reversal potential of $\sim 0 \mathrm{mV}$ (data not shown), demonstrating that it is a glutamatergic EPSC (Fig. $1 B$ ). Stimulation of DAergic axons also activated NMDA receptor (NMDAR) currents in all neurons exhibiting a peak amplitude of $23 \pm 3 \mathrm{pA}$ (range, $10-46 \mathrm{pA}$ ), a rise time of $6.5 \pm 0.5 \mathrm{~ms}$, and monoexponential decay with a time constant of $77 \pm 9 \mathrm{~ms}\left(V_{\mathrm{h}}=\right.$ $+50 \mathrm{mV}, n=26$ ) (Fig. 1C). The glutamatergic responses were accompanied by DA release detected with FSCV, as described below (Fig. $2 D, E$ ). Potentially DA-mediated nonglutamatergic postsynaptic responses were not observed (data not shown). Glutamatergic EPSCs were observed throughout both the shell and the core of the nucleus accumbens. In five of seven tested SPNs 
exhibiting large-amplitude EPSCs, singlepulse stimulation was sufficient to elicit action potentials at membrane potentials approximating the typical in vivo "up" state of these neurons (Fig. $1 D$ ).

One potential concern with the optogenetic method is that the relatively slow and partially $\mathrm{Ca}^{2+}$ ion-mediated ChR2 currents may induce nonphysiological activity patterns in the stimulated axons or recruit abnormal release mechanisms in presynaptic terminals. To control for this, we first examined the firing responses of single DAergic axons during optical stimulation using cell-attached patch recording $(n=3)$ from blebs formed at cut ends of these axons identified by enhanced YFP fluorescence while simultaneously recording light-evoked EPSCs in nearby SPNs (Fig. 2A). DAergic axons were able to follow the stimuli faithfully, firing single time-locked action potentials to each light pulse, and never responded with bursts (Fig. $2 A$; see Fig. $4 A$ ). The spikes exhibited precise coincidence with the onset or rising phase of EPSCs recorded in nearby neurons (Fig. $2 A$; see Fig. $4 A$ ). Second, we determined whether the postsynaptic responses were induced by propagating action potentials in DAergic axons. Application of tetrodotoxin (TTX; $1 \mu \mathrm{M})$ abolished the glutamatergic EPSCs, demonstrating that they were dependent on $\mathrm{Na}_{\mathrm{v}}$ channels (Fig. $2 \mathrm{~B}$ ). To directly test whether these responses were elicited by propagating action potentials, we examined the effect of increasing the distance between the recorded neuron and the site of focal laser stimulation on the onset latency of the EPSC (Fig. 2C). The latency of the EPSCs increased monotonically with the distance between the stimulation and recording sites (range, 6-14 ms over $\sim 100-1460 \mu \mathrm{m}$ ), demonstrating the involvement of propagating spikes $(n=4)$ (Fig. $2 C$ ). Finally, to examine whether the probability or short-term plasticity of release from DAergic terminals was altered by optical stimulation we used FSCV to compare the rate of extracellular DA accumulation during optical and conventional electrical train stimulation of DAergic axons (five pulses at $10 \mathrm{~Hz}$ ) (Fig. 2D,E) (see Materials and Methods for further explanation). The identity of the released substance as DA was determined based on the identical potentials of the oxidation and reduction peaks and the overall waveforms of voltammograms obtained in a DA standard and in the slices during optical or electrical stimulation (Fig. 2D). The ratios of DA concentrations measured at $300 \mathrm{~ms}$ vs those measured at $100 \mathrm{~ms}$ after stimulus onset using electrical stimulation (accumbens core, $2.1 \pm 0.2$; shell, $2.7 \pm 0.3 ; n=9$ ) were not different from the corresponding values obtained using optical stimuli (accumbens core, $2.4 \pm 0.2 ;$ shell, $2.4 \pm 0.4 ; n=9 ; p>$ $0.28, t$ test), indicating that the release properties were not meatrace).
B

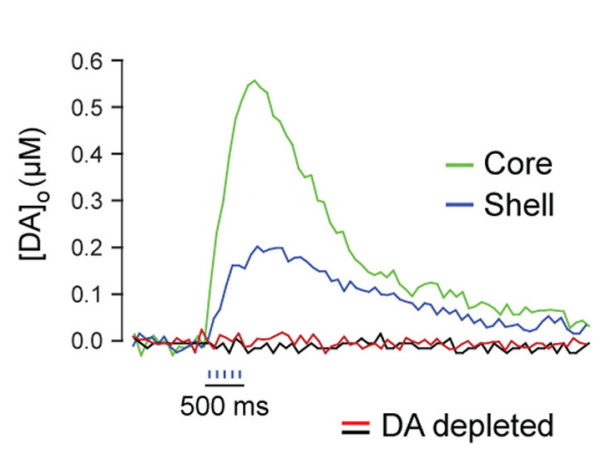

- CNQX

Figure 3. Glutamatergic responses are independent of DAergic signaling. $\boldsymbol{A}$, The optically elicited EPSC (control, green trace) is not altered by the blockade of $\mathrm{D}_{1}$ and $\mathrm{D}_{2}$ DA receptors with $\mathrm{SCH} 21390(\mathrm{SCH} ; 10 \mu \mathrm{m})$ and sulpiride (SUL; $5 \mu \mathrm{m}$, blue). $\boldsymbol{B}$, Pharmatrace) of the accumbens in a control animal is absent in slices from a DA-depleted animal (red and black traces). C, Normal AMPA receptor-mediated EPSCs are elicited optically (blue bar) in an SPN after complete DA depletion (averaged response, green trace; individual responses, gray traces). The response is reversibly blocked by an AMPAR antagonist (CNQX, $10 \mu \mathrm{m}$, red trace; wash, blue

A

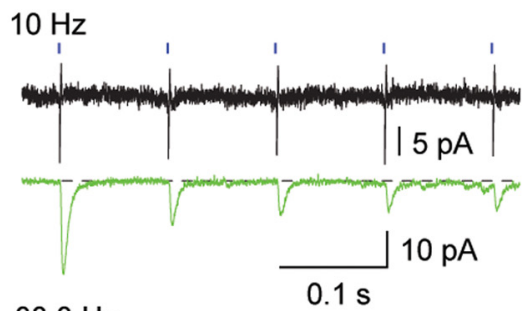

$33.3 \mathrm{~Hz}$

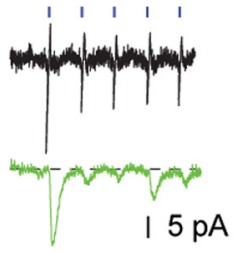

Figure 4. Glutamatergic responses of SPNs to a behaviorally relevant pattern of activity of DAergic axons. $\boldsymbol{A}$, Simultaneous cell-attached patch recording from a DAergic axon (black traces) and somatic whole-cell recording from a nearby SPN (green traces) during optical train stimulation demonstrate reliable axonal firing at 10 or $33.3 \mathrm{~Hz}$ (blue ticks, five pulses, 5 ms duration each). Evoked EPSCs are coincident with the axonal spikes. $\boldsymbol{B}$, Optically evoked action potential bursts (blue ticks; $33.3 \mathrm{~Hz}$; three increasing peak current during the stimulus. Inset shows higher time resolution of the first $160 \mathrm{~ms}$ of the response (total current in green; (NQX, $10 \mu \mathrm{m}$, in blue)

surably altered by optical stimulation. The similarity of the optically and electrically evoked DA transients are illustrated for the accumbens shell in Figure $3 E$; the results obtained in the core were nearly identical (data not shown). Together, these experiments demonstrate that the optically elicited synaptic release is both qualitatively and quantitatively representative of release elicited by the physiological activity of these neurons.

Next, to address the question of whether the glutamatergic EPSCs are generated directly by the release of glutamate from DAergic axon terminals or are secondary to released DA we first tested the effect of pharmacological blockade of $D_{1}$ and $D_{2}$ family DA receptors with coapplication of the selective antagonists SCH21390 $(10 \mu \mathrm{M})$ and sulpiride $(5 \mu \mathrm{M})$ (Fig. 3A). Blockade of 
DA receptors had no detectable effect on the glutamatergic EPSC (Fig. 3A). Since DA can interact with several non-DAergic receptors and might have other nonconventional effects, we also tested the effects of complete depletion of the cytoplasmic and vesicular DA pools with in vivo pretreatment and subsequent continuous in vitro application of the vesicular monoamine transporter blocker reserpine and the TH inhibitor $\alpha$-methyl-para-tyrosine. The effectiveness of this treatment was verified using FSCV in the nucleus accumbens (Fig. 3B). Postsynaptic glutamatergic EPSCs with qualitative properties indistinguishable from those in normal slices were present in all SPNs tested after depletion of DA (amplitude, $28 \pm 1.4 \mathrm{pA} ; n=4$ ) (Fig. 4C). Based on these observations and on the precise coincidence of presynaptic action potential firing and the onset of the postsynaptic EPSCs described above (Figs. 2A,4A), we conclude that the postsynaptic EPSCs are monosynaptic and originate from glutamate released directly from DAergic axons.

Critical to the function of DA neurons in reward processing is their firing of brief population bursts during the presentation of primary rewards and reward-predicting cues, which in rodents typically consists of individual neurons firing two to three action potentials at 30-40 Hz (Hyland et al., 2002; Pan et al., 2005). Because of the functional importance of this population response, we sought to estimate the postsynaptic glutamatergic response of accumbens neurons to a pattern of activation of DAergic axons that simulates this response. To ensure that burst responses could be reliably elicited in presynaptic axons, we obtained cell-attached recordings from DAergic axons in the accumbens during optical train stimulation consisting of up to five light pulses of 2 or $5 \mathrm{~ms}$ duration delivered at 10 or $33.3 \mathrm{~Hz}$. The axons could faithfully follow stimulation at these frequencies $(n=3)$ (Fig. 4A). Next, we recorded EPSCs in accumbens neurons in response to $33.3 \mathrm{~Hz}$ train stimuli (three pulses, $n=9$ ) under the same conditions. Postsynaptic responses consisted of coincident trains of AMPAR- and NMDAR-mediated EPSCs. AMPAR EPSCs exhibited significant short-term depression (third/first EPSC $\left._{\mathrm{AMPA}}=0.44 \pm 0.1 ; \mathrm{Vm}=-70 \mathrm{mV}\right)($ Fig. $4 \mathrm{~B})$, while the NMDA receptor-mediated currents, which also showed short-term depression, exhibited significant temporal summation, resulting in an increasing peak NMDAR current amplitude throughout the train $\left(\mathrm{EPSC}_{\mathrm{NMDA}}\right.$ at third/first stimulus $=1.35 \pm$ 0.35; $\mathrm{Vm}=+50 \mathrm{mV}$ ) (Fig. $4 B$ ).

\section{Discussion}

The present study is the first direct demonstration that mesolimbic DAergic neurons release glutamate under physiological conditions and confirms the conclusions of earlier cell culture (Sulzer et al., 1998; Bourque and Trudeau, 2000; Joyce and Rayport, 2000; Sulzer and Rayport, 2000) and slice (Chuhma et al., 2004, 2009) experiments, which were consistent with an early in vivo intracellular recording study (Wilson et al., 1982).

Interestingly, the variable and limited short-term depression or short-term facilitation of glutamatergic responses elicited in the nucleus accumbens using electrical stimulation of the VTA (Chuhma et al., 2004, 2009) is markedly different from the consistent and pronounced short-term depression observed in our experiments. This observation suggests that the nucleus accumbens receives more than one type of glutamatergic afferents from the VTA, including non-DAergic projections exhibiting distinctive properties of short-term plasticity.

Although these differences could have resulted from unequal involvement of axonal branch point failure when proximal versus distal stimulation is used, this is highly unlikely given the com- plete absence of branch point failures observed in DAergic neurons in antidromic stimulation studies in vivo (Tepper et al., 1984). The differences also could reflect late postnatal developmental differences (Tepper et al., 1990).

Glutamatergic signaling by the mesolimbic DAergic projection is of significant interest because it represents a novel mechanism for reward signaling with sufficiently fast kinetics to decode the information represented in the reward-related transient firing responses of DA neurons. Although the glutamatergic EPSCs elicited by burst activation of the DAergic input (Fig. $4 B$ ) cannot directly account for the typical reward-related responses of accumbens neurons (Carelli and Deadwyler, 1994), these synaptic responses may be well suited to briefly synchronize the activity of accumbens SPNs. Another important role of glutamate release from DA neurons may be the regulation of the plasticity of cortical and limbic glutamatergic inputs to the nucleus accumbens. Trans-synaptic regulation of synaptic plasticity may be mediated by synaptic cross talk predicted by the proximity of axospinous glutamatergic and DAergic synapses $(<1 \mu \mathrm{m})$ (Moss and Bolam, 2008), which is comparable to the experimentally and theoretically estimated permissive range of extrasynaptic activation of NMDA receptors (Rusakov et al., 1999). In addition, DAergic synapses on the neck of dendritic spines forming a triadic arrangement with non-DAergic axospinous synapses (Somogyi et al., 1981; Freund et al., 1984) may allow intracellular trans-synaptic communication, which, in addition to previously suggested analogous DAergic interactions (Freund et al., 1984; Goldman-Rakic et al., 1989), may regulate the long-term plasticity of cortical and limbic inputs through glutamatergic signaling, including NMDA receptor-mediated intracellular calcium signals.

\section{References}

Bérubé-Carrière N, Riad M, Dal Bo G, Lévesque D, Trudeau LE, Descarries L (2009) The dual dopamine-glutamate phenotype of growing mesencephalic neurons regresses in mature rat brain. J Comp Neurol 517:873-891.

Bourque MJ, Trudeau LE (2000) GDNF enhances the synaptic efficacy of dopaminergic neurons in culture. Eur J Neurosci 12:3172-3180.

Carelli RM, Deadwyler SA (1994) A comparison of nucleus accumbens neuronal firing patterns during cocaine self-administration and water reinforcement in rats. J Neurosci 14:7735-7746.

Chen BT, Rice EM (2001) Novel $\mathrm{Ca}^{2+}$ dependence and time course of somatodendritic dopamine release: substantia nigra versus striatum. J Neurosci 21:7841-7847.

Chuhma N, Zhang H, Masson J, Zhuang X, Sulzer D, Hen R, Rayport S (2004) Dopamine neurons mediate a fast excitatory signal via their glutamatergic synapses. J Neurosci 24:972-981.

Chuhma N, Choi WY, Mingote S, Rayport S (2009) Dopamine neuron glutamate cotransmission: frequency-dependent modulation in the mesoventromedial projection. Neuroscience 164:1068-1083.

Freund TF, Powell JF, Smith AD (1984) Tyrosine hydroxylase-immunoreactive boutons in synaptic contact with identified striatonigral neurons, with particular reference to dendritic spines. Neuroscience 13:11891215.

Goldman-Rakic PS, Leranth C, Williams SM, Mons N, Geffard M (1989) Dopamine synaptic complex with pyramidal neurons in primate cerebral cortex. Proc Natl Acad Sci U S A 86:9015-9019.

Gras C, Herzog E, Bellenchi GC, Bernard V, Ravassard P, Pohl M, Gasnier B, Giros B, El Mestikawy S (2002) A third vesicular glutamate transporter expressed by cholinergic and serotoninergic neurons. J Neurosci 22:5442-5451.

Hyland BI, Reynolds JN, Hay J, Perk CG, Miller R (2002) Firing modes of midbrain dopamine cells in the freely moving rat. Neuroscience 114:475-492.

Joyce MP, Rayport S (2000) Mesoaccumbens dopamine neuron synapses reconstructed in vitro are glutamatergic. Neuroscience 99:445-456. 
Juraska JM, Wilson CJ, Groves PM (1977) The substantia nigra of the rat: a Golgi study. J Comp Neurol 172:585-600.

Mendez JA, Bourque MJ, Dal Bo G, Bourdeau ML, Danik M, Williams S, Lacaille JC, Trudeau LE (2008) Developmental and target-dependent regulation of vesicular glutamate transporter expression by dopamine neurons. J Neurosci 28:6309-6318.

Moss J, Bolam JP (2008) A dopaminergic axon lattice in the striatum and its relationship with cortical and thalamic terminals. J Neurosci 28:1122111230.

Pan WX, Schmidt R, Wickens JR, Hyland BI (2005) Dopamine cells respond to predicted events during classical conditioning: evidence for eligibility traces in the reward-learning network. J Neurosci 25:6235-6242.

Patel JC, Witkovsky P, Avshalumov MV, Rice EM (2009) Mobilization of calcium from intracellular stores facilitates somatodendritic dopamine release. J Neurosci 29:6568-6579.

Rusakov DA, Kullmann DM, Stewart MG (1999) Hippocampal synapses: do they talk to their neighbours? Trends Neurosci 22:382-388.

Somogyi P, Bolam JP, Smith AD (1981) Monosynaptic cortical input and local axon collaterals of identified striatonigral neurons. A light and electron microscopic study using the Golgi-peroxidase transport-degeneration procedure. J Comp Neurol 195:567-584.

Sullivan MA, Chen H, Morikawa H (2008) Recurrent inhibitory network among striatal cholinergic interneurons. J Neurosci 28:8682-8690.
Sulzer D, Rayport S (2000) Dale's principle and glutamate corelease from ventral midbrain dopamine neurons. Amino Acids 19:45-52.

Sulzer D, Joyce MP, Lin L, Geldwert D, Haber SN, Hattori T, Rayport S (1998) Dopamine neurons make glutamatergic synapses in vitro. J Neurosci 18:4588-4602.

Tepper JM, Young SJ, Groves PM (1984) Autoreceptor-mediated changes in dopaminergic terminal excitability: effects of increases in impulse flow. Brain Res 309:309-316.

Tepper JM, Sawyer SF, Groves PM (1987) Electrophysiologically identified nigral dopaminergic neurons intracellularly labeled with HRP: lightmicroscopic analysis. J Neurosci 7:2794-2806.

Tepper JM, Trent F, Nakamura S (1990) Postnatal development of the electrical activity of rat nigrostriatal dopaminerglc neurons. Dev Brain Res 54:21-33.

Tsai HC, Zhang F, Adamantidis A, Stuber GD, Bonci A, de Lecea L, Deisseroth $\mathrm{K}$ (2009) Phasic firing in dopaminergic neurons is sufficient for behavioral conditioning. Science 324:1080-1084.

Wilson CJ, Chang HT, Kitai ST (1982) Origins of postsynaptic potentials evoked in identified rat neostriatal neurons by stimulation in substantia nigra. Exp Brain Res 45:157-167.

Yamaguchi T, Sheen W, Morales M (2007) Glutamatergic neurons are present in the rat ventral tegmental area. Eur J Neurosci 25:106-118. 